\title{
Clinical Profile and Outcome of Critically Ill Post COVID-19 Patients in The Non COVID-19 Intensive Care Unit of a Tertiary Hospital in Indonesia
}

\author{
Jilientasia Godrace Lilihata $^{1 *}$, Bambang Pujo Semedi ${ }^{1}$, Lucky Andriyanto ${ }^{1}$ \\ ${ }^{1}$ Department of Anesthesiology and Intensive Therapy, Faculty of Medicine Universitas Airlangga, Dr. Soetomo General Hospital, \\ Surabaya \\ *Email: jilientasia@gmail.com
}

\begin{abstract}
Background: Survival rates of severe COVID-19 has increased. Despite that COVID-19 appears to leave sequelae, hence the need of critical care and mortality after prolonged treatment. There were not many studies which investigated critically ill post COVID-19 patients. This study aimed to analyze clinical profiles and their relationship to outcomes of critically ill post-COVID-19 patients in a regular (non COVID-19) ICU at a tertiary hospital in Indonesia.

Materials and methods: This single-centre, retrospective study was conducted at Dr. Soetomo General Hospital, Indonesia between March 1st and October 31st, 2021 with total sampling method. Subjects were post COVID-19 patients from the COVID-19 ICU and ward who have been declared RT-PCR negative twice but require further intensive care in the regular ICU. Bivariate analysis was performed using chi-square, Mann-Whitney, and independent t-test. Multivariate analysis was carried out by logistic regression. The results were considered significant when $\mathrm{p}<0.05$.

Results: The study included 76 critically ill patients who had completed their COVID-19 treatment in the COVID-19 ward or COVID-19 ICU and required further intensive care. Bivariate analysis shwed obesity (p 0.047), CNS disease (p 0.038), complications in the form of sepsis ( $\mathrm{p} 0.002$ ), secondary infection (p 0.001), and the presence of MDRO (p 0.017) were significantly associated with mortality. Multivariate analysis showed risk factors of mortality were complications that were AKI, cardiac complications, secondary infection, sepsis and non COVID-19 ICU LOS.

Conclusion: Complications AKI, cardiac complications, sepsis, and secondary infection were risk factors for mortality in critically ill post COVID-19 patients in the ICU.
\end{abstract}

Keywords:post COVID-19; critically ill, ICU, outcome

\section{Intoduction}

COVID-19 has affected many countries since it was declared a pandemic in March 2020. Various efforts have been made to reduce the spread and mortality of COVID-19, including vaccination campaigns and periodic social restrictions, as well as studies on the best treatment methods, particularly for patients with severe and critical COVID-19 manifestations. Overall survival rates increased in critically ill COVID-19 patients in 2021 [1]. However the increasing survival rate has not solved the problem because it turns out that COVID-19 can leave sequelae, the need for Intensive Care Unit (ICU) care, and even death after prolonged treatment or post-COVID-19 readmission [2, 3].

There is no precise data regarding complications or profiles and outcomes for critically ill post COVID-19 patients, especially in Indonesia. This study aims to analyze clinical profiles and their relationship to outcomes of critically ill post COVID-19 patients in a regular (non COVID-19) ICU at a tertiary hospital in Indonesia. 


\section{Materials and Methods}

This single-centre, retrospective study was conducted at Dr. Soetomo General Hospital in Surabaya, East Java, Indonesia. The study was conducted with approval of the local ethics committee. The research period was between March 1st, 2020, and October 31st, 2021, with total sampling method. As many as 76 post COVID-19 hospitalized patients from the COVID-19 ICU and COVID-19 ward have been declared RT-PCR negative twice but require further intensive care. These patients were transferred to a standard ICU (non COVID-19) room for further treatment. Demographic data, including comorbidities, length of stay (LOS) in the non COVID-19 ICU, complications during treatment, and outcomes, were obtained from patients' medical records.

The Kolmogorov-Smirnoff normality test was first used before performing descriptive analysis. The data were tabulated, the number and percentage displayed in the mean \pm SD (standard deviation) for normally distributed data and the median $(\min -\max )$ for non-normally distributed data. Bivariate analysis was performed using chi-square test for categorical variables and Mann-Whitney test (non-parametric) or independent t-test (non-parametric) for continuous variables. Multivariate analysis was carried out by logistic regression to find risk factors for mortality. The results were considered significant when $\mathrm{p}<0.05$.

\section{Results}

The study included 76 critically ill patients who had completed their COVID-19 treatment in the COVID19 ward or COVID-19 ICU and required further intensive care. Median age was 51.5 years, $55.3 \%$ of the patients were men. The oldest patient was 76 years old and the youngest was 19 years old. A total of 18 (23.7 $\%$ ) critically ill post COVID-19 patients had no comorbidities. The rest of them had at least one comorbidity, with Diabetes Mellitus being the most common (43.4\%) one. The most common complications during non COVID-19 ICU treatment were respiratory complications, including difficulty weaning from the ventilator, diaphragmatic muscle weakness, low $\mathrm{PaO} 2 / \mathrm{FiO} 2$ ratio with or without pneumonia, and pneumothorax. A total of 51 patients $(67.1 \%)$ were alive when discharged from the non COVID-19 ICU. Table 1 displays data on patient characteristics.

The bivariate analysis of the outcome revealed that obesity (BMI $>30 \mathrm{~kg} / \mathrm{m} 2)(\mathrm{p} 0.047)$, Centra Nervous System (CNS) disease ( $\mathrm{p}$ 0.038), treatment complications in the form of sepsis (p 0.002), secondary infection (p 0.001), and the presence of Multidrug Resistance Organisms (MDRO) (p 0.017) were significantly associated with the outcome of post COVID-19 critical patients in non COVID-19 ICU as seen in table 2. In this study, sepsis could be described as a condition that has persisted since the COVID-19 ICU treatment period or as a secondary infection in a non COVID-19 ICU room in critically ill post COVID-19 patients.

Secondary infections were recorded in $30.3 \%$ of the study's population, where bacteria were detected as pathogens in blood cultures, urine cultures, or sputum cultures of critically ill post COVID-19 patients taken after 48 hours of treatment in the non COVID-19 ICU and accompanied by infection symptoms. MDRO organisms were discovered in $25 \%$ of the study population. Acinetobacter baumanii was the most frequent MDRO pathogen, followed by Klebsiella pneumoniae and Pseudomonas aeruginosa. There are Staphylococcus epidermidis and Candida spp. as non MDRO potential pathogens. Based on culture results, most infections emerged from the patient's sputum.

The multivariate test discovered five factors that influence the patient's mortality outcome, including the incidence of acute kidney injury [4]. Cardiac disorders such as arrhythmia, myocardial infarction, or heart failure, secondary infection in non COVID-19 ICU, sepsis, and length of stay in non COVID-19 ICU rooms. Patients who were critically ill after COVID-19 and had complication Acute Kidney Injury (AKI) (p 0.002) had a 16-fold higher risk of mortality than patients who did not have AKI with OR $=16.16$ (95 percent 
confidence interval $=2.84-91.78)$. Non COVID-19 ICU LOS was negatively related to mortality with OR = 0.89 (95 percent confidence interval $=0.82-0.97$ ). Table 3 shows the details of the multivariate analysis results.

Table 1. Patients characteristics.

\begin{tabular}{|c|c|c|c|}
\hline Variables $($ mean \pm SD) & $\mathbf{N}(\mathbf{7 6})$ & Percentages $(\%)$ & Median (min - max) \\
\hline \multicolumn{4}{|l|}{ 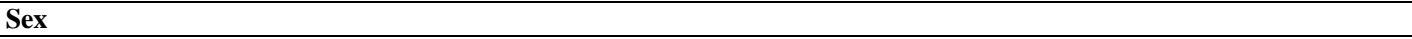 } \\
\hline Male & 42 & 55.3 & \\
\hline Female & 34 & 44.7 & \\
\hline BMI $(26.02 \pm 5.69)$ & & & $24.80(18.37-61.68)$ \\
\hline \multicolumn{4}{|l|}{ Comorbidities } \\
\hline 1 comorbidity & 29 & 38.2 & \\
\hline$>1$ comorbidities & 29 & 38.2 & \\
\hline Diabetes Mellitus & 33 & 43.4 & \\
\hline Hypertension & 23 & 30.3 & \\
\hline Chronic Kidney Disease & 9 & 11.8 & \\
\hline Obesity & 8 & 10.5 & \\
\hline Chronic Liver Disease & 7 & 9.2 & \\
\hline Central Nervous System Disease & 5 & 6.6 & \\
\hline Cardiac Disease & 5 & 6.6 & \\
\hline Chronic Lung Disease & 3 & 3.9 & \\
\hline Autoimune Disease & 3 & 3.9 & \\
\hline \multicolumn{4}{|l|}{ Treatment Complications } \\
\hline Respiratory Complication & 55 & 72.4 & \\
\hline Sepsis & 43 & 56.6 & \\
\hline Secondary Infection & 23 & 30.3 & \\
\hline Acute Kidney Injury & 22 & 28.9 & \\
\hline Acute Liver Dysfunction & 21 & 27.6 & \\
\hline Cardiac Complications & 12 & 15.8 & \\
\hline \multicolumn{4}{|l|}{ Length of stay } \\
\hline Non COVID-19 ICU $(8.55 \pm 7.52)$ & & & $7(1-43)$ \\
\hline \multicolumn{4}{|l|}{ Outcomes } \\
\hline Survived & 51 & 67.1 & \\
\hline Dead & 25 & 32.9 & \\
\hline
\end{tabular}

\section{Discussion}

Indonesia is one of the countries that still limits patient care in the COVID-19 treatment room based on RTPCR results and clinical symptoms. Due to the limited public facilities for treating critical COVID-19 patients, the Indonesian government has established a standard operating procedure (SOP) for acute COVID-19 patients treated in the isolation ICU. If the patient's RT-PCR results have been negative for two examinations, but the patient still requires intensive care, the patient will be transferred to the regular non COVID-19 ICU so that the isolation ICU can be used for new patients who are critical and had a positive RT-PCR test result. There are not many studies that analyze critically ill patients after COVID-19, but the findings in this study were consistent with other studies.

In this study, we discovered that most patients who still required further critical care post COVID-19 infection were men, most common comorbidities were diabetes mellitus, hypertension, chronic kidney 
disease, obesity. The non COVID-19 ICU mortality rate was 32.9\%. Previous research that compared the characteristics of each wave of the COVID-19 pandemic had similar results, most of the patients were men and the most common comorbidities were hypertension, obesity, and diabetes mellitus. High Flow Nasal Cannula (HFNC) use increased during the second and third periods of the COVID-19 pandemic [5]. Another study with more than 1,000 participants obtained similar results, showing that more than half of the patients requiring hospital treatment were men, and nearly $20 \%$ needed ICU care. The ICU mortality outcome was $30.6 \%[6]$.

Table 2. Bivariate analysis.

\begin{tabular}{|c|c|c|c|}
\hline Variables & Survived $(\mathrm{N}=51)$ & Died $(\mathrm{N}=25)$ & $\mathbf{p}$ \\
\hline \multicolumn{3}{|l|}{ Sex } & \multirow{3}{*}{1.000} \\
\hline Male & 28 & 14 & \\
\hline Female & 23 & 11 & \\
\hline Age & $47.98 \pm 13.43$ (years) & $54.36 \pm 12.46$ (years) & 0.050 \\
\hline BMI & $24.22(18.37-37.65)$ & $25.39(21.48-61.68)$ & 0.558 \\
\hline \multicolumn{3}{|l|}{ Comorbidities } & \multirow{4}{*}{0.209} \\
\hline No comorbidities & 15 & 3 & \\
\hline 1 comorbidity & 19 & 10 & \\
\hline$>1$ comorbidities & 17 & 12 & \\
\hline Diabetes Mellitus & $18 / 51$ & $15 / 25$ & 0.073 \\
\hline Hypertension & $14 / 51$ & $9 / 25$ & 0.620 \\
\hline Chronic Kidney Disease & $5 / 51$ & $4 / 25$ & 0.465 \\
\hline Obesity & $8 / 51$ & $0 / 25$ & $0.047 *$ \\
\hline Chronic Liver Disease & $4 / 51$ & $3 / 25$ & 0.678 \\
\hline Central Nervous System Disease & $1 / 51$ & $4 / 25$ & $0.038^{*}$ \\
\hline Cardiac Disease & $2 / 51$ & $3 / 25$ & 0.324 \\
\hline Chronic Lung Disease & $3 / 51$ & $0 / 25$ & 0.209 \\
\hline Autoimune Disease & $3 / 51$ & $0 / 25$ & 0.547 \\
\hline \multicolumn{4}{|l|}{ Treatment Complications } \\
\hline Respiratory Complication & $36 / 51$ & $19 / 25$ & 0.824 \\
\hline Sepsis & $22 / 51$ & $21 / 25$ & $0.002 *$ \\
\hline Secondary Infection & $8 / 51$ & $15 / 25$ & $0.001 *$ \\
\hline Acute Kidney Injury & $7 / 51$ & $15 / 25$ & 1.000 \\
\hline Acute Liver Dysfunction & $14 / 51$ & $7 / 25$ & 1.000 \\
\hline Cardiac Complications & $5 / 51$ & $7 / 25$ & 0.052 \\
\hline $\begin{array}{l}\text { Presence of Multi-drug Resistance } \\
\text { Organisms }\end{array}$ & $8 / 51$ & $11 / 25$ & $0.017 *$ \\
\hline \multicolumn{4}{|l|}{ Length of stay } \\
\hline Non COVID-19 ICU & $7(1-43)$ & $6(1-26)$ & 0.239 \\
\hline
\end{tabular}

Table 3. Multivariate analysis.

\begin{tabular}{ccccc}
\hline Variables & B & p & OR & CI 95\% \\
\hline Acute Kidney Injury & 2.78 & 0.002 & 16.16 & $1.84-91.78$ \\
\hline Cardiac Complications & 2.73 & 0.023 & 15.29 & $1.45-160.98$ \\
\hline Secondary Infection & 2.38 & 0.007 & 10.83 & $1.94-60.45$ \\
\hline Sepsis & 2.89 & 0.024 & 10.83 & $1.94-60.45$ \\
\hline Non COVID-19 ICU LOS & -0.11 & 0.008 & 0.89 & $0.82-0.97$ \\
\hline
\end{tabular}


This study population consisted of critically ill patients who survived the COVID-19 infection period (twice negative RT-PCR) but required post COVID-19 intensive care. We observed a similar age range to other studies that noted intensive care for post COVID-19 patients [2, 3]. Older age is at risk for more comorbidities and there is an increased risk of mortality at age $>50$ years old in COVID-19 patients [7]. However, in this study age, BMI, and gender were not significant predictors of a bad outcome in critically ill post COVID-19 patients.

Similar to COVID-19 patients in China who also used negative RT-PCR as a conditions in the treatment of post COVID-19 [8, 9], this study showed that non COVID-19 ICU LOS tended to be shorter for non survivors. Respiratory complications $(72.4 \%)$ or difficulty weaning and secondary infection $(30.3 \%)$ were the most common complications during treatment. Another study found that the highest number of deaths occurred in patients with an ICU LOS of 1 to 10 days, while patients with more than ten days in the ICU had a $65 \%$ ICU survival. Ventilator dependency, secondary infection, and coma may cause prolonged ICU stays [10].

The most common comorbidity in the COVID-19 population in Indonesia is hypertension, followed by diabetes mellitus and cardiovascular comorbidities. These comorbidities were also associated with worse outcome and longer LOS [5, 11]. Comorbidity was significantly associated with an increased risk of mortality. Kidney disease had the highest risk, followed by cerebral vascular disease, heart disease, lung disease, diabetes, hypertension, and cancer. The chronic liver disease did not significantly increase the risk of mortality in post COVID-19 patients [7].

Obesity related to a pro-inflammatory condition that linked to insulin resistance, metabolic syndrome, and prothrombotic disorders, worsening the body's immune response to pathogens, including COVID-19. It was one of the most common comorbidities and a predictor of poor outcomes in COVID-19 research [5, 12, 13]. In this study, statistical analysis revealed that comorbidities such as obesity and CNS diseases, including stroke and epilepsy, were significantly associated with outcomes but were not risk factors for mortality.

In this study population, AKI was the most significant risk factor for mortality, followed by cardiovascular disorders, secondary infections, and sepsis. AKI is one of the clinical complications associated with mortality, depending on the stage. The higher the stage of AKI, the greater the risk of mortality. Patients with AKI are also at risk for developing Chronic Kidney Disease (CKD) in the future and Acute on Chronic Kidney Disease (ACKD) may worsen the progression of CKD in these patients. For this reason, it is emphasized that AKI should be prevented in various ways [14]. Another study in Indonesia also found that AKI was significantly associated with mortality in ICU patients $[15,16]$.

The most common cardiac complications discovered in this study were arrhythmias and cardiac ischemia with or without heart disease comorbidity. Another study found arrhythmias to be the most common complication and a risk factor for mortality in the care of patients in the ICU in a population of COVID-19 and non COVID-19 patients with Community Acquired Pneumonia (CAP). Conditions like atrial fibrillation or ventricular tachycardia can trigger other complications such as stroke, heart failure, and even death. Every occurrence of arrhythmia needs to be found and treated for its cause [17, 18].

Ventilator Associated Pneumonia (VAP) was the most common complication of COVID-19 patients and a predictor of COVID-19 ICU mortality [5]. Some of the causes and complications of intensive care are sepsis and secondary infections. The most common causes of sepsis are respiratory tract and abdominal infections, nosocomial infections related to catheter and ventilator usage during ICU care. Death due to sepsis is more common in developing countries than in developed countries. The length of stay in the ICU also increased compared to patients who did not have sepsis. Acinetobacter baumanii infection increases with mechanical ventilation and predicts poor outcomes in ICU patients [19, 20]. 
This study's limitations included the small number of samples and the lack of analysis on therapeutic variables that may influence the outcome. Due to the sheer limitation, viewers must interpret some findings with caution. The results may differ if the study were conducted on a larger scale or in multiple centers.

\section{Conclusion}

Comorbidities like obesity, CNS disease, sepsis, and MDRO secondary infection affected the patient's outcome. Complications AKI, cardiac complications, sepsis, and secondary infection were risk factors for mortality in critically ill post COVID-19 patients in the ICU.

\section{Conflict of Interests}

None.

\section{Acknowledgements}

None.

\section{References}

1. Bateson ML, McPeake JM. Critical care survival rates in COVID-19 patients improved as the first wave of the pandemic developed. Evid Based Nurs. 2021;0(0):5-6.

2. Subramaniam A, Lim ZJ, Reddy MP, Shekar K. Systematic review and meta- analysis of the characteristics and outcomes of readmitted COVID-19 survivors. Internal Medicine Journal. 2021;51(11):1773-80.

3. Donnelly JP, Wang XQ, Iwashyna TJ, Prescott HC. Readmission and Death after Initial Hospital Discharge among Patients with COVID-19 in a Large Multihospital System. JAMA - J Am Med Assoc. 2021;325(3):304-6.

4. Kellum JA, Lameire N, Aspelin P, Barsoum RS, Burdmann EA. Kidney Disease: Improving Global Outcomes (KDIGO) Acute Kidney Injury Work Group. KDIGO Clinical Practice Guideline for Acute Kidney Injury. Kidney inter, Suppl 2012 [Internet]. 2012;(2):1-138. Available from: https://kdigo.org/guidelines/acute-kidney-injury/

5. Carbonell R, Urgelés S, Rodríguez A, Bodí M, Martín-Loeches I, Solé-Violán J, et al. Mortality comparison between the first and second/third waves among 3,795 critical COVID-19 patients with pneumonia admitted to the ICU: A multicentre retrospective cohort study. Lancet Reg Heal - Eur. 2021;11.

6. Pouw N, Maat J Van De, Veerman K, Oever J, Janssen N, Abbink E, et al. Clinical characteristics and outcomes of 952 hospitalized COVID-19 patients in The Netherlands : A retrospective cohort study. Plos One. 2021;183:1-15.

7. Biswas M, Rahaman S, Biswas TK, Haque Z, Ibrahim B. Association of Sex, Age, and Comorbidities with Mortality in COVID-19 Patients: A Systematic Review and Meta-Analysis. Intervirology. 2021;64(1):36-47.

8. Rees EM, Nightingale ES, Jafari Y, Waterlow NR, Clifford S, Carl CA, et al. COVID-19 length of hospital stay: A systematic review and data synthesis. BMC Med. 2020;18(1).

9. Yadav AK, Ghosh S, Kotwal A, Kaushik SK, Bobdey S, Sahu R, et al. Seroconversion among COVID-19 patients admitted in a dedicated COVID hospital: A longitudinal prospective study of 1000 patients. Med J Armed Forces India. 2020;7(Suppl. 2):379-84.

10. Rimachi R, Vincent J, Brimioulle S. survival and quality of life after prolonged intensive care unit stay. Anaesthesia and Intensive Care. 2007;35(1).

11. Karyono DR, Wicaksana AL. Current prevalence, characteristics, and comorbidities of patients with COVID-19 in Indonesia. J Community Empower Heal. 2020;3(2):77.

12. Thomson RJ, Hunter J, Dutton J, Schneider J, Khosravi M, Casement A, et al. Clinical characteristics and outcomes of critically ill patients with COVID-19 admitted to an intensive care unit in London: A prospective observational cohort study. Plos One. 2020;38:1-16.

13. Bello-Chavolla OY, Bahena-Lopez JP, Antonio-Villa NE, Vargas A, Gonzalez-Diaz A, Márquez-Salinas A, et al. Predicting mortality due to SARS-CoV-2: A mechanistic score relating obesity and diabetes to COVID-19 outcomes in Mexico. The 
Journal of Clinical Endocrinology \& Metabolism. 2020;105(8):2752-61.

14. Jamme M, Legrand M, Geri G. Outcome of acute kidney injury: how to make a difference? Ann Intensive Care. 2021;11(1).

15. Hidayat H, Pradian E, Kestriani ND. Angka Kejadian, Lama Rawat, dan Mortalitas Pasien Acute Kidney Injury di ICU RSUP Dr. Hasan Sadikin Bandung. J Anestesi Perioper. 2020;8(2):108-18.

16. Rasyid F, Semedi BP, Utariani A, Aswin T. Relationship Between Renal Angina Index and D-Dimer Improvement Towards Acute Kidney Injury in Covid-19 Patients in Special Isolation Room of Dr. Soetomo Hospital Surabaya. Int J Sci Adv. 2021;2(2):164-7.

17. Martinot M, Eyriey M, Gravier S, Bonijoly T, Kayser D, Ion C, et al. Predictors of mortality, ICU hospitalization, and extrapulmonary complications in COVID-19 patients. Infect Dis Now. 2021;51(6):518-25.

18. Cilli A, Cakin O, Aksoy E, Kargin F, Adiguzel N, Karakurt Z, et al. Acute cardiac events in severe community-acquired pneumonia: A multicenter study. Clin Respir J. 2018;12(7):2212-9.

19. Sakr Y, Jaschinski U, Wittebole X, Szakmany T, Lipman J, Ñamendys-silva SA, et al. Sepsis in Intensive Care Unit Patients : Worldwide Data From the Intensive Care over Nations Audit. Open Forum Infect Dis. 2018;1-9.

20. Suranadi IW, Nengah N, Fatmawati D, Aryabiantara IW. Acinetobacter baumannii is an opportunistic pathogen as an MDRO in ICU. Bali Journal of Anesthesiology. 2019;3(2):150-3. 